\title{
Autosomal Recessive Osteogenesis Imperfecta Caused by a Novel Homozygous COL1A2 Mutation
}

\section{Costantini, Alice}

2018-09

Costantini , A , Tournis , S , Kämpe , A , Ul Ain , N , Taylan , F, Doulgeraki , A \& Mäkitie , O 2018 , ' Autosomal Recessive Osteogenesis Imperfecta Caused by a Novel Homozygous COL1A2 Mutation ' , Calcified Tissue International , vol. 103 , no. 3 , pp. 353-358 . https://doi.org/10.1007/s00223-01

http://hdl.handle.net/10138/312192

https://doi.org/10.1007/s00223-018-0414-4

acceptedVersion

Downloaded from Helda, University of Helsinki institutional repository.

This is an electronic reprint of the original article.

This reprint may differ from the original in pagination and typographic detail.

Please cite the original version. 
1 Autosomal recessive osteogenesis imperfecta caused by a novel homozygous COL1A2

2 mutation

3 Alice Costantini ${ }^{1}$, Symeon Tournis ${ }^{2}$, Anders Kämpe ${ }^{1}$, Noor Ul Ain ${ }^{1,3}$, Fulya Taylan ${ }^{1}$, Artemis

4 Doulgeraki ${ }^{4}$, Outi Mäkitie ${ }^{1,5,6,7}$

5

$6{ }^{1}$ Department of Molecular Medicine and Surgery and Center for Molecular Medicine, Karolinska Institutet,

7 Stockholm, Sweden

$8{ }^{2}$ Laboratory for Research of the Musculoskeletal System 'Th. Garofalidis', KAT Hospital, National and

9 Kapodistrian University of Athens, School of Medicine, Athens, Greece

$10{ }^{3}$ School of Biological Sciences, University of the Punjab, Lahore, Pakistan

$11{ }^{4}$ Department of Bone and Mineral Metabolism, Institute of Child Health, "Aghia Sophia" Children's Hospital,

12 Athens, Greece

$13{ }^{5}$ Folkhälsan Institute of Genetics, Helsinki, Finland

$14{ }^{6}$ Children's Hospital, University of Helsinki and Helsinki University Hospital, Helsinki, Finland

$15 \quad{ }^{7}$ Department of Clinical Genetics, Karolinska University Hospital, Stockholm, Sweden

16

17 Corresponding author: Alice Costantini, Department of Molecular Medicine and

18 Surgery, Center for Molecular Medicine (CMM) L8:02, Karolinska University

19 Hospital Solna, 17176 Stockholm, Sweden

20 E-mail address: alice.costantini@ki.se

21 Phone: +46720207058

22 ORCID: 0000-0003-1408-9272

23

24 Running title: Homozygous $\operatorname{COL} 1 A 2$ mutations in OI

25

26 Supplemental data are included in the submission. 
Osteogenesis imperfecta (OI) is a skeletal dysplasia characterized by brittle bones and extra-skeletal manifestations. The disease phenotype varies greatly. Most commonly, OI arises from monoallelic mutations in one of the two genes encoding type I collagen, COL1A1 and COL1A2 and is inherited as an autosomal dominant trait. Here we describe a consanguineous family with autosomal recessive OI caused by a novel homozygous glycine substitution in COL1A2, NM_000089.3: c.604G>A, p.(Gly202Ser), detected by whole-genome sequencing. The index patient is a 31-year-old Greek woman with severe skeletal fragility. She had mild short stature, low bone mineral density of the lumbar spine and blue sclerae. She had sustained multiple long bone and vertebral

37 fractures since childhood and had been treated with bisphosphonates for several years. She also had an affected sister with similar clinical manifestations. Interestingly, the parents and

39 one sister, all carriers of the COL1A2 glycine mutation, did not have manifestations of OI.

40 In summary, we report on autosomal recessive Ol caused by a homozygous glycine to 41 serine substitution in $\operatorname{COL} 1 A 2$, leading to severe skeletal fragility. The mutation carriers 42 lacked OI manifestations. This family further expands the complex genetic spectrum of OI 43 and underscores the importance of genetic evaluation for correct genetic counselling.

45 Key words: autosomal recessive; type I collagen; osteogenesis imperfecta; BMD; fracture 


\section{INTRODUCTION}

47 Osteogenesis imperfecta (OI) is a skeletal dysplasia characterized by bone fragility and low

48 bone mineral density (BMD). In 9 out of 10 cases, $\mathrm{Ol}$ is caused by dominantly inherited

49 heterozygous mutations in one of the two genes encoding the $\alpha 1$ and $\alpha 2$ chains of type I

50 collagen (COL1A1 and COL1A2, respectively). The remaining $10 \%$ of cases are due to

51 autosomal recessive and X-linked mutations in one of the several other recently identified

52 Ol genes that are involved in post-translational modification of type I collagen, in osteoblast

53 function, or in other aspects of bone metabolism [1, 2].

54 Biallelic mutations in COL1A2 have been described in a few consanguineous families, in

55 which Ol is inherited as an autosomal recessive trait [3-5]. Depending on the location of the

56 mutation, homozygous mutations in COL1A2 can also give rise to Ehlers-Danlos syndrome $57 \quad[6-8]$.

58 Here we report on a novel biallelic mutation in COL1A2 causing moderately severe OI in two

59 Greek women born to consanguineous parents.

60

\section{2. SUBJECTS AND METHODS}

\subsection{Subjects}

63 As part of an ongoing research project on genetic causes of inherited skeletal diseases we

64 recruited a Greek-Cypriot family with skeletal fragility. The index patient had traits

65 suspicious of $\mathrm{OI}$ and her consanguineous parents ( $2^{\text {nd }}$ cousins) were healthy. The patient had three sisters of whom one also had a history of multiple fractures.

\subsection{Methods}

68

Clinical data were collected from hospital records. In order to investigate the genetic cause

69 of the skeletal disease in our index patient we performed whole-genome sequencing (WGS) 
on all family members (index patient, her parents and her three sisters). Detailed

71 information about this method can be found in Supplemental Appendix S1.

72 Variant validation was performed using Sanger sequencing.

\section{RESULTS}

\subsection{Clinical report}

A Greek-Cypriot woman, born in 1986, was first evaluated for potential OI when she was 27 years old. She was born to healthy consanguineous parents ( $2^{\text {nd }}$ cousins) at term and had no antenatal or perinatal history of note. Apart from her mild short stature, she had blue sclerae, while dentition was normal (Table 1).

At age 8-11 yrs, she had suffered multiple low-energy fractures (bilateral Colle's, forearm, clavicle, ankle, pubic bone and at least three vertebral fractures). Thereafter, she had no further skeletal complications until age $21 \mathrm{yrs}$, when she sustained a new radius fracture. After bisphosphonate treatment (oral risedronate for 1 year and thereafter 3 yearly courses of $5 \mathrm{mg}$ intravenous zoledronic acid), at age $25 \mathrm{yrs}$ bone mineral density (BMD) at the lumbar spine (LS) was compatible with osteoporosis (Table 1). Two years later, during a respiratory tract infection, she suffered multiple rib fractures and a comminuted right acetabular fracture after a fall from standing height (Fig. 1). She underwent internal fixation, complicated by pulmonary embolism. At that time, she received one dose (60 $\mathrm{mg}$ ) of denosumab s.c. Three months later, at $28 \mathrm{yrs}$, she suffered a fracture of the left femoral neck. At this time some spinal changes were also detected and confirmed recently by

91 vertebral fracture assessment (VFA) (Fig.1). Due to this stormy clinical course, nine months 
most recent evaluation at $31 \mathrm{yrs}$, she had not experienced any further fractures and BMD

94 measurements were within normal limits (Table 1).

95 The patient's family history was suggestive of a recessive form of primary osteoporosis. Her 96 parents, who were $2^{\text {nd }}$ cousins, were free of skeletal fragility. The mother had osteopenia on 97 the DXA scan but her fracture history was negative. The father's BMD was normal. The 98 index patient had three older sisters, two free from skeletal manifestations and one affected by excessive bone fragility and Ol features, including blue sclerae and dentinogenesis imperfecta (Fig. 2A; Table 1). This affected sister had sustained a tibial 101 fracture and multiple rib fractures between 11 and 12 yrs of age and a scaphoid fracture of 102 the wrist at the age of $15 \mathrm{yrs}$. She also had three healthy children.

\subsection{Genetic investigations}

104 WGS in the six family members identified two autosomal recessive variants with potentially 105 damaging effect: 1) a novel single nucleotide variant in exon 13 of COL1A2, NM_000089.3: 106 c.604G>A, p.(Gly202Ser) (Fig. 2B) and 2) a 4-nucleotides intronic indel, TAAA, in RP11107 682N22.1, which encodes a long intergenic non-coding RNA (lincRNA). Both variants were 108 identified in homozygosity in the index patient and her affected sister and in heterozygosity 109 in the parents and one healthy sister. Surprisingly, all the mutation carriers were healthy. 110 The other healthy sister was not a mutation carrier.

111 The molecular function of the lincRNA is unknown. However, monoallelic mutations 112 affecting the Gly-Xaa-Yaa repeats in the triple helix of type I collagen are the most common 113 cause of OI. For this reason, we regarded the COL1A2 variant, inherited with an atypical 114 autosomal recessive pattern of inheritance, as the cause of the disease in our index patient 115 and her affected sister. This variant is also determined as pathogenic according to the 116 ACMG guidelines and was confirmed by Sanger sequencing (Fig. 2C) [9]. 


\section{DISCUSSION}

119 In this report we describe a novel autosomal recessive COL1A2 mutation, c.604G>A, 120 p.(Gly202Ser), identified in a Greek consanguineous family. The clinical features of the 121 index patient and her affected sister were compatible with moderately severe OI whereas 122 the mutation carriers lacked OI manifestations.

123 While biallelic mutations of the $\alpha 1(I)$ chain are probably lethal, homozygous mutations of 124 the $\alpha 2(I)$ chain have been described in a handful of families [3-5, 10-12]. Similar to the case 125 reported by De Paepe et al, our index patient harbours a homozygous mutation leading to 126 glycine to serine substitution in the $\alpha 2(I)$ chain [4]. She presents with several OI hallmarks 127 including mild short stature, blue sclerae, low BMD and recurrent fractures since childhood. 128 Interestingly, her fracture pattern is unusual, since it includes pelvis and acetabular 129 fractures, which are uncommon in OI. Similarly, the affected sister had sustained multiple 130 rib fractures and a scaphoid fracture of the wrist, both of which are not typical OI fractures. 131 Although there is no evidence of a similar fracture pattern in other patients with biallelic 132 COL1A2 mutations, this finding may reflect atypical bone brittleness due to a complete lack 133 of normal type I collagen. Considering that type I collagen is the most abundant component 134 in bone and cartilage, it is likely that complete depletion of the wild-type protein causes a 135 severe skeletal phenotype with a fracture pattern that differs from the common autosomal 136 dominant type I collagen-related OI. On the other hand, despite the autosomal recessive 137 inheritance pattern, these clinical features were in line with type I collagen-related OI 138 rather than the several autosomal recessive forms of OI [13].

139 In contrast to the mutation carriers described by De Paepe et al. who had mild skeletal 140 manifestations, the mutation carriers reported here lack signs and symptoms of skeletal 
141 fragility or other OI manifestations [4]. One of them (the mother) has osteopenia but other

142 carriers have normal BMD and all have a negative fracture history. It is surprising that a 143 heterozygous glycine substitution within the triple-helical region does not result in any 144 impaired skeletal phenotype $[1,3,5,6]$. This suggests that the severity of the Ol phenotype 145 depends on location of the glycine substitution within the helical region. In fact, other 146 patients with a glycine to serine substitutions in the $\alpha 2(I)$ chain have been described as 147 having mild to moderate OI [4, 12]. However, the mutation we identified locates closer to 148 the N-terminal $\alpha 2(I)$ chain than the other reported cases. In fact, it affects glycine 202 and 149 mutations in the first $200 \mathrm{~N}$-terminal amino acids only slightly influence the triple helix 150 stability $[1,14]$. This could explain why our carriers do not show any Ol manifestation. 151 Furthermore, it is possible that a more detailed bone tissue assessment with histology and 152 histomorphometry could have detected mild abnormalities in the heterozygotes, but in any 153 case there are no clinically obvious manifestations of OI.

154 In summary, we diagnosed a challenging case of a patient with autosomal recessive Ol due 155 to a novel homozygous COL1A2 mutation, NM_000089.3:c.604G>A, p.(Gly202Ser). 156 Interestingly, this mutation leads to Ol features only in homozygosity. Our finding 157 underscores the complexity of $\mathrm{Ol}$ and the variability in its genetic causes and inheritance 158 pattern. Further, our study emphasizes the importance of a genetic diagnosis in all patients 159 with OI to ensure correct genetic counselling.

\section{ACKNOWLEDGMENTS}

162 We would like to thank the Swedish Research Council for the financial support and the 163 Science for Life Laboratory (SciLifeLab) for supplying bioinformatics support. 
166 All authors declare no conflict of interest.

167 The study protocol was approved by the Institutional Ethics Committee and the 168 experiments were performed in accordance to the Declaration of Helsinki.

169 A written informed consent was obtained from each participant before inclusion into the 170 study.

\section{CONTRIBUTIONS}

173 Study design: AC, AD, OM. Study conduct: AC, ST, AD. Data collection: AC, ST, AD, OM. Data 174 analysis: AC. Data interpretation: AC, ST, AK, NUA, FT, AD, OM. Drafting manuscript: AC, ST, 175 AD, OM. Revision of manuscript content: all authors. Approval of final version of 176 manuscript: all authors. Responsibility for the integrity of the data: all authors. 
180 1. Marini JC, Forlino A, Bachinger HP, Bishop NJ, Byers PH, Paepe A, Fassier F,

10. Byers PH, Pyott SM (2012) Recessively inherited forms of osteogenesis imperfecta. Annual review of genetics 46:475-497

11. Schnieke A, Harbers K, Jaenisch R (1983) Embryonic lethal mutation in mice induced by retrovirus insertion into the á1(I) collagen gene. Nature 304:315320

12. Marini JC, Lewis MB, Wang Q, Chen KJ, Orrison BM (1993) Serine for Glycine Substitutions in Type I Collagen in Two Cases of Type IV Osteogenesis Imperfecta (OI). The Journal of biological chemistry 268:2667-2673

13. Van Dijk FS, Sillence DO (2014) Osteogenesis imperfecta: clinical diagnosis, nomenclature and severity assessment. American journal of medical genetics. Part A 164A:1470-1481

14. Marini JC, Forlino A, Cabral WA, Barnes AM, San Antonio JD, Milgrom S, Hyland JC, Korkko J, Prockop DJ, De Paepe A, Coucke P, Symoens S, Glorieux FH, Roughley PJ, Lund AM, Kuurila-Svahn K, Hartikka H, Cohn DH, Krakow D, Mottes M, Schwarze U, Chen D, Yang K, Kuslich C, Troendle J, Dalgleish R, Byers PH (2007) Consortium for osteogenesis imperfecta mutations in the helical domain of type I collagen: regions rich in lethal mutations align with collagen binding sites for integrins and proteoglycans. Human mutation 28:209-221 


\begin{tabular}{|c|c|c|c|c|c|c|c|c|c|c|c|}
\hline & $\begin{array}{l}\overline{0} \\
\stackrel{0}{z} \\
\stackrel{\Delta}{0} \\
0\end{array}$ & 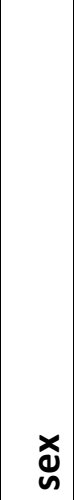 & 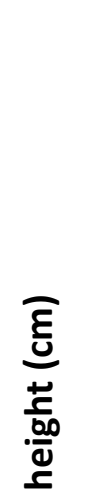 & 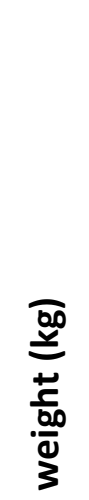 & 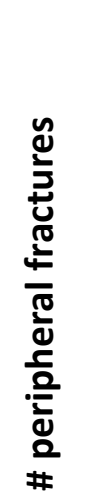 & 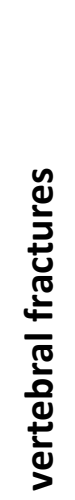 & 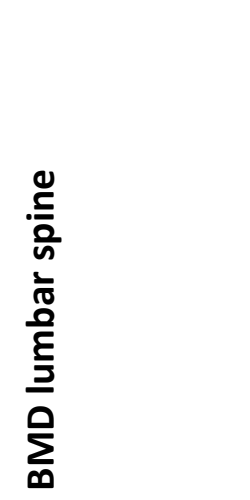 & 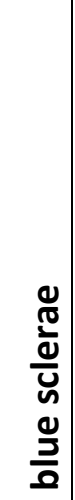 & 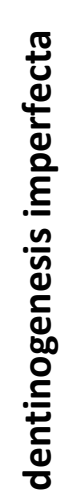 & $\begin{array}{l}\tilde{y} \\
\stackrel{0}{\pi} \\
\frac{1}{0} \\
\frac{1}{0} \\
\frac{0}{0} \\
\frac{0}{0} \\
\frac{0}{0}\end{array}$ & 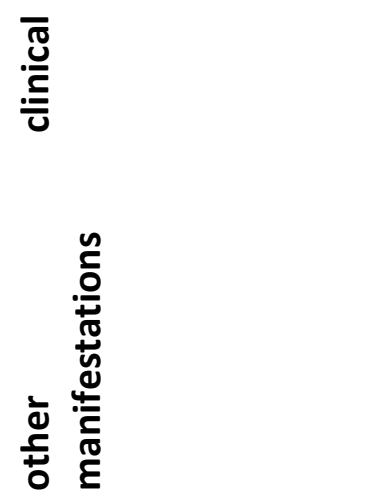 \\
\hline $\begin{array}{l}\text { father } \\
(\mathrm{I}-1)\end{array}$ & 68 & $M$ & 172 & 100 & 0 & 0 & -1.0 (T-score) & - & - & - & hypertension \\
\hline $\begin{array}{l}\text { mother } \\
(1-2)\end{array}$ & 59 & $\mathrm{~F}$ & 158 & 90 & 0 & 0 & -2.1 (T-score) & - & - & - & $\begin{array}{l}\text { type } 2 \text { diabetes, } \\
\text { arterial hypertension }\end{array}$ \\
\hline $\begin{array}{l}\text { index } \\
\text { (II-4) }\end{array}$ & 31 & $\mathrm{~F}$ & 154 & 48 & $>10$ & 3 & -2.2 (Z-score) & + & - & + & - \\
\hline $\begin{array}{l}\text { sibling1 } \\
\text { (II-1) }\end{array}$ & 42 & $\mathrm{~F}$ & 157 & 58 & 0 & 0 & -0.2 (Z-score) & - & - & - & - \\
\hline $\begin{array}{l}\text { sibling } 2 \\
\text { (II-2) }\end{array}$ & 41 & $F$ & 163 & 98 & 0 & 0 & 1.2 (Z-score) & - & - & - & Graves' disease \\
\hline $\begin{array}{l}\text { sibling } 3 \\
\text { (II-3) }\end{array}$ & 38 & $\mathrm{~F}$ & 155 & 80 & $>4$ & NA & -1.3 (Z-score) & + & + & - & - \\
\hline
\end{tabular}




\section{Figure legends}

\section{Figure 1}

Lateral radiograph of the thoracolumbar spine showing A) A mild biconcave deformity of L1 vertebra. The vertebral fractures sustained during childhood are not clearly evident in the X-ray and VFA, probably due to vertebral body reshaping during growth. B) Internal fixation of the pelvis. C) VFA of the same patient; mild biconcave deformity of L1 vertebra. D) Anteroposterior radiograph of the pelvis showing internal fixation of a right acetabular fracture.

\section{Figure 2}

A) Pedigree of the family. B) Screenshot from the Integrative Genomic Visualization (IGV) program showing the WGS reads mapped to reference genome GRCh37 at the COL1A2 locus where the c.604G>A, p.(Gly202Ser) mutation locates. Roman numerals refer to the pedigree in Fig. 2A. C) Sanger sequencing validation. Arrows indicate the mutation. 
Figures

249 Figure 1
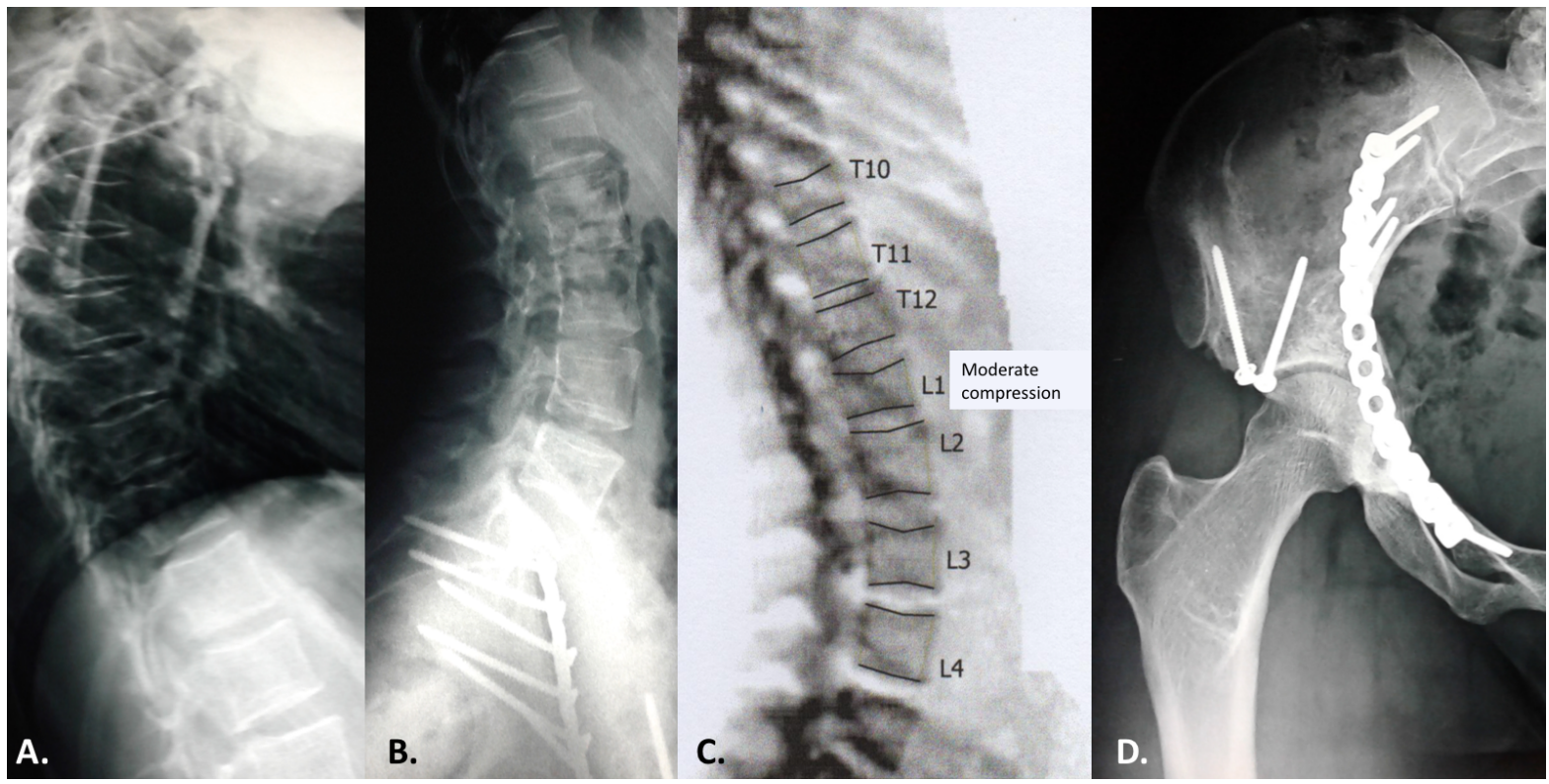

Figure 2
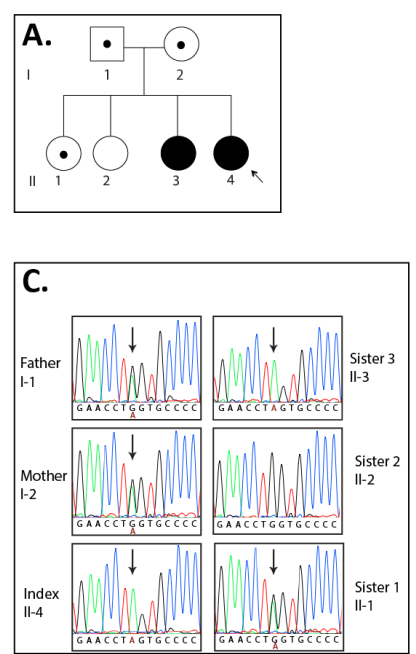

B.

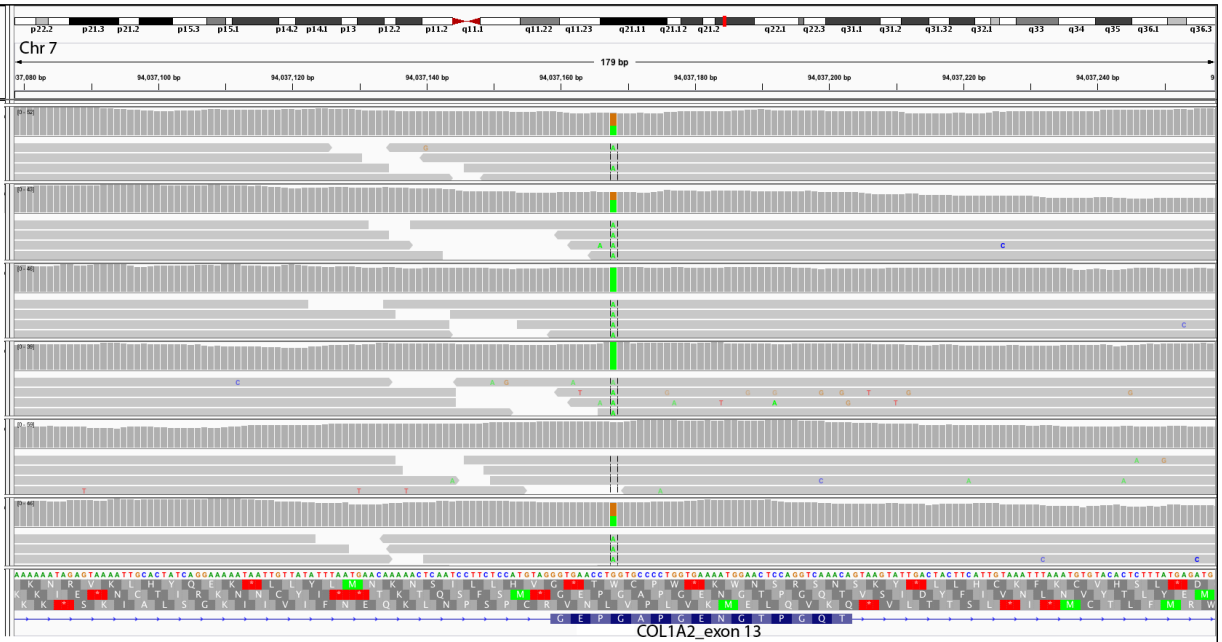

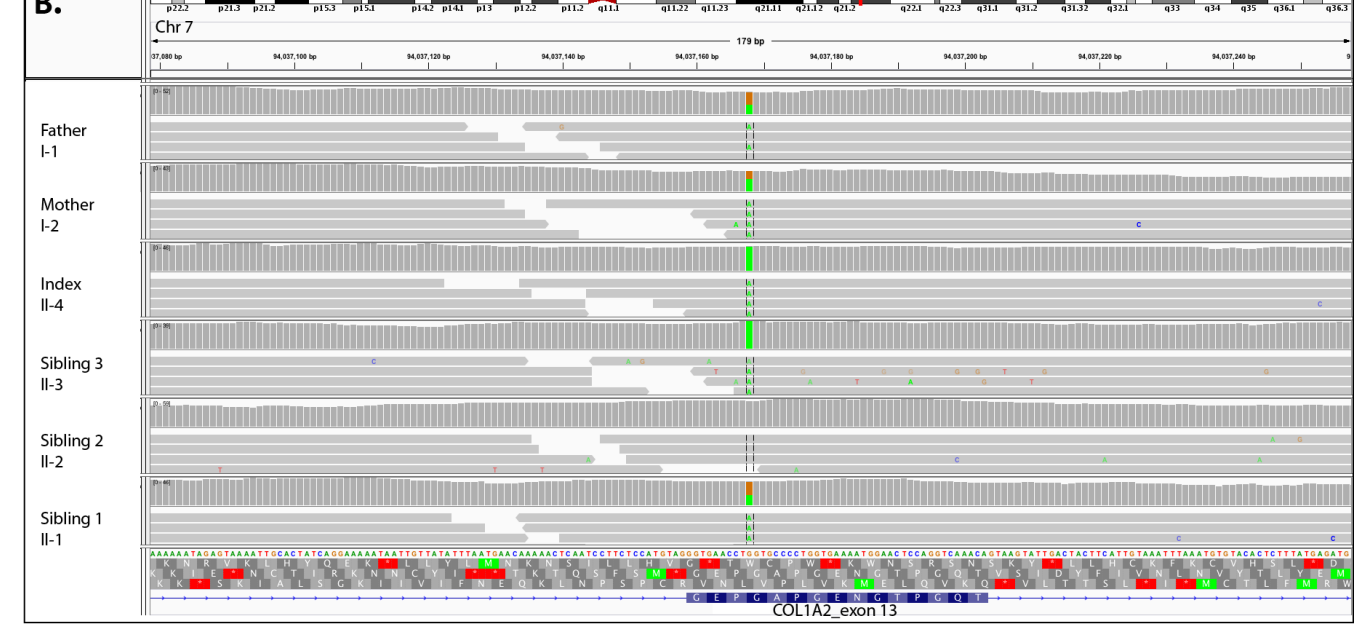

